\title{
Editorial
}

\section{Evidence in Urologic- and Pelvic-Surgery Research: Finding the IDEAL Way of Reporting}

\author{
H. Gerullis, ${ }^{1}$ D. Barski, ${ }^{2}$ P. U. Malmström, ${ }^{3}$ X. Sun, ${ }^{4}$ and T. H. Ecke ${ }^{5}$ \\ ${ }^{1}$ University Hospital for Urology, Klinikum Oldenburg, School of Medicine and Health Sciences, \\ Carl von Ossietzky University Oldenburg, Oldenburg, Germany \\ ${ }^{2}$ Department of Urology, Lukas Hospital, Neuss, Germany \\ ${ }^{3}$ Department of Urology, Uppsala University, Uppsala, Sweden \\ ${ }^{4}$ University of Chengdu, Chengdu, China \\ ${ }^{5}$ Department of Urology, HELIOS Hospital, Bad Saarow, Germany
}

Correspondence should be addressed to H. Gerullis; holger.gerullis@gmx.net

Received 5 March 2017; Accepted 5 March 2017

Copyright (C) 2017 H. Gerullis et al. This is an open access article distributed under the Creative Commons Attribution License, which permits unrestricted use, distribution, and reproduction in any medium, provided the original work is properly cited.

In 2009, the Idea, Development, Exploration, Assessment, Long-Term Follow-Up (IDEAL) Collaboration, an international, Oxford-based group of surgeons and methodologists, suggested a template of clear recommendations to define the fundamental stages of surgical innovations and related research [1-3]. IDEAL provides a framework for the evaluation of surgical innovations comparable to the existing standards for drug development. Ever since, the IDEAL recommendations have increasingly been applied in surgical research and reporting.

This special issue has been introduced with the aim of offering the possibility of publishing research results and particularly discuss them according to IDEAL to urologists and researchers connected to the field of urosurgery, urogynecology, surgery, and pelvic surgery.

While editing this special issue we have learned that the awareness towards the IDEAL recommendations is not as developed as one could hope. Although IDEAL has been applied in several prospective research projects and even shown applicable when retrospectively reporting the status of a surgical method or innovation it has not been used by the majority of submissions to this special issue. However, not labelling an innovative surgical or diagnostic method does not diminish its value as the recommendations have existed for 7 years only. This special issue aimed to contribute in divulging IDEAL and in encouraging surgeons and scientists to use these recommendations when reporting their innovations. As a conclusion it can be stated that the awareness of IDEAL needs to increase among both researchers and reviewers.

\section{Acknowledgments}

The editors thank all submitting authors for their efforts and time spent for each manuscript. The lead editor would like to thank all editors for the time spent in reviewing, assigning reviews, and commenting on submitted manuscripts. As editorial team, we hope that this special issue will prove useful to surgeons and researchers involved in the field of pelvic surgery, urology and urogynecology. We hope that it would help to better apply and integrate the IDEAL recommendations into planning, conducting, and reporting of surgical research.

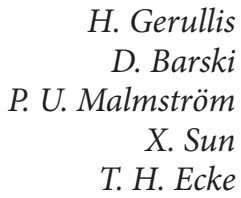

H. Gerullis Därö

T. H. Ecke 


\section{References}

[1] J. S. Barkun, J. K. Aronson, L. S. Feldman et al., "Evaluation and stages of surgical innovations," The Lancet, vol. 374, no. 9695, pp. 1089-1096, 2009.

[2] P. L. Ergina, J. A. Cook, J. M. Blazeby et al., "Challenges in evaluating surgical innovation," The Lancet, vol. 374, no. 9695, pp. 1097-1104, 2009.

[3] P. McCulloch, D. G. Altman, W. B. Campbell et al., "No surgical innovation without evaluation: the IDEAL recommendations," The Lancet, vol. 374, no. 9695, pp. 1105-1112, 2009. 


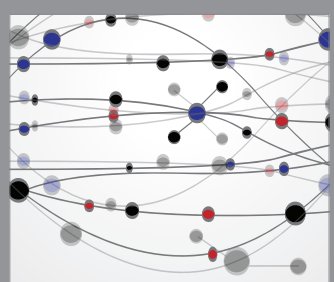

The Scientific World Journal
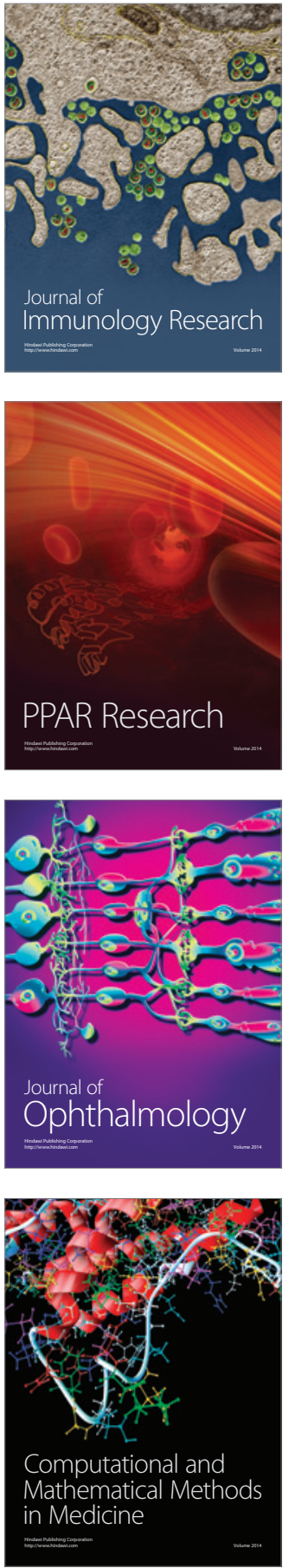

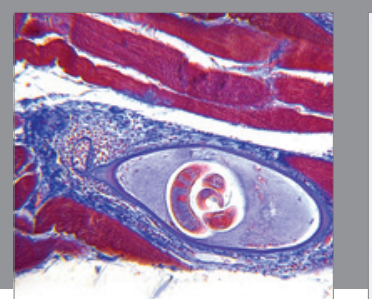

Gastroenterology Research and Practice
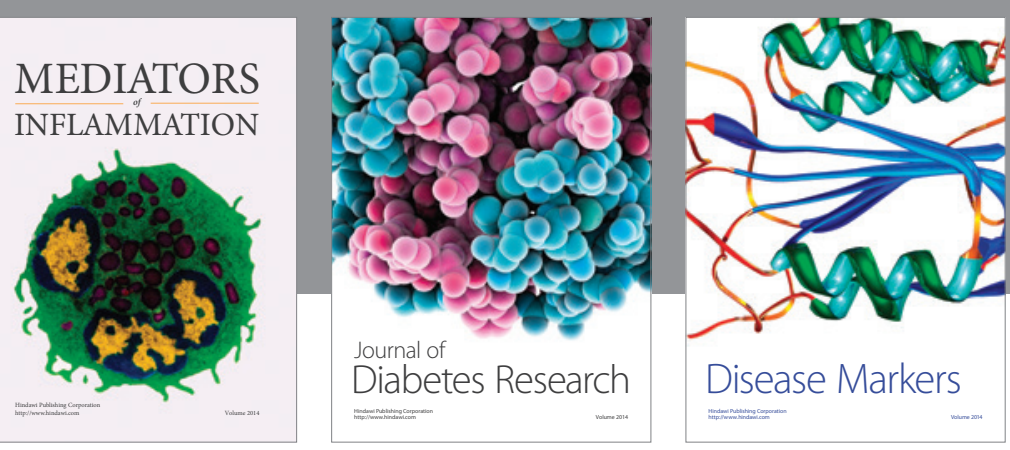

Disease Markers

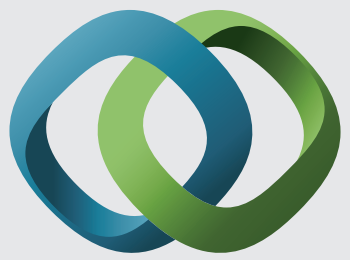

\section{Hindawi}

Submit your manuscripts at

https://www.hindawi.com
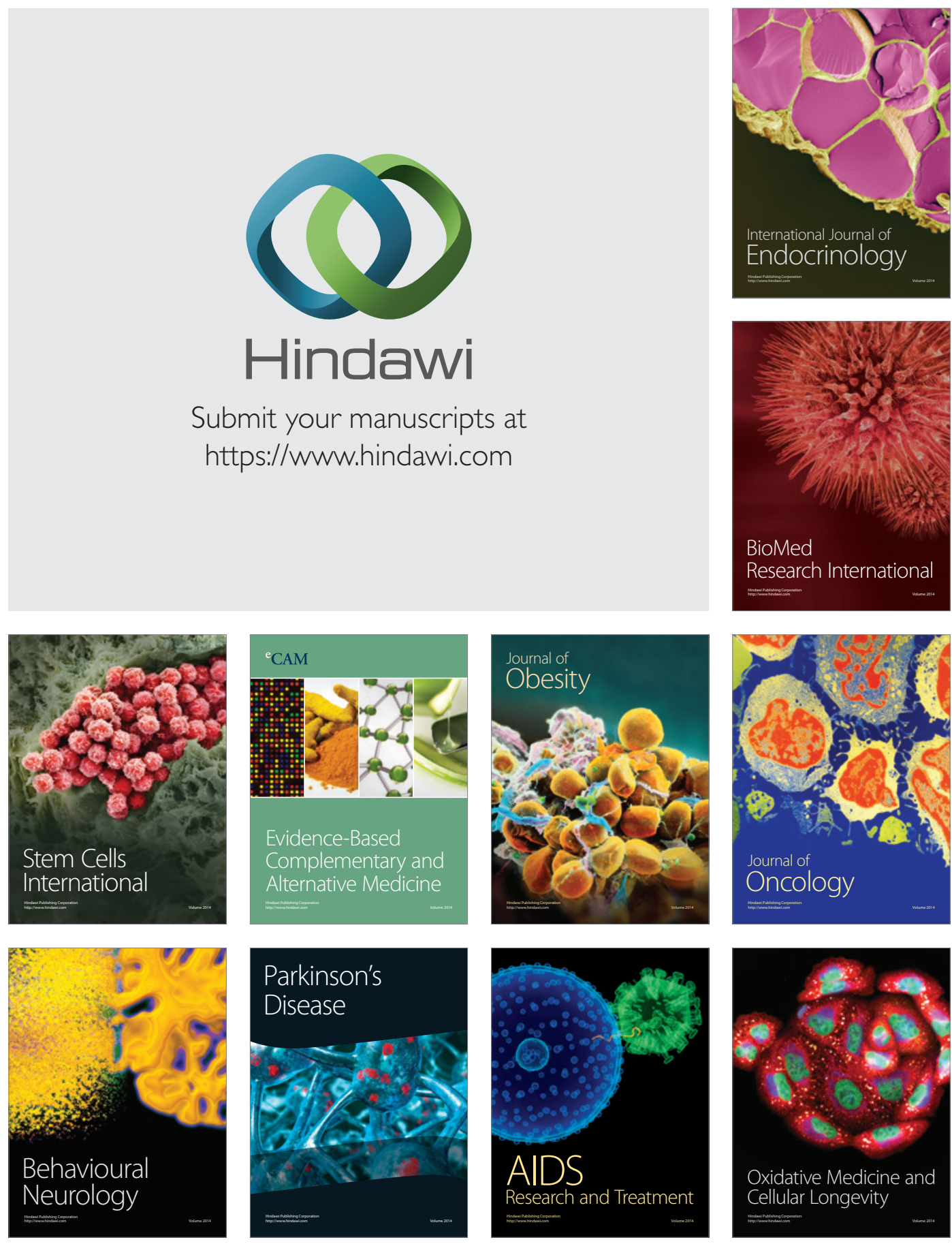